\title{
Achieving the Gaussian Rate-Distortion Function by Prediction
}

\author{
Ram Zamir, Yuval Kochman and Uri Erez Dept. Electrical Engineering-Systems, Tel Aviv University
}

\begin{abstract}
The "water-filling" solution for the quadratic ratedistortion function of a stationary Gaussian source is given in terms of its power spectrum. This formula naturally lends itself to a frequency domain "test-channel" realization. We provide an alternative time-domain realization for the rate-distortion function, based on linear prediction. The predictive test-channel has some interesting implications, including the optimality at all distortion levels of pre/post filtered vector-quantized differential pulse code modulation (DPCM), and a duality relationship with decisionfeedback equalization (DFE) for inter-symbol interference (ISI) channels.
\end{abstract}

Keywords: Test channel, water-filling, pre/post-filtering, DPCM, Shannon lower bound, ECDQ, directed-information, equalization, MMSE estimation, decision feedback.

\section{INTRODUCTION}

The water-filling solution for the quadratic rate-distortion function $R(D)$ of a stationary Gaussian source is given in terms of the spectrum of the source. Similarly, the capacity $C$ of a power-constrained ISI channel with Gaussian noise is given by a water-filling solution relative to the effective noise spectrum. Both these formulas amount to limiting values of mutual-information between vectors in the frequency domain. In contrast, linear prediction along the time domain can translate these vector mutual-information quantities into scalar ones. Indeed, for capacity, Cioffi et al [4] showed that $C$ is equal to the scalar mutual-information over a slicer embedded in a decision-feedback noise-prediction loop.

We show that a parallel result holds for the rate-distortion function: $R(D)$ is equal to the scalar mutual-information over an additive white Gaussian noise (AWGN) channel embedded in a source prediction loop, as shown in Figure 1 This result implies that $R(D)$ can essentially be realized in a sequential manner (as will be clarified later), and it joins other observations regarding the role of minimum mean-square error (MMSE) estimation in successive encoding and decoding of Gaussian channels and sources [7], [6], [3].

\section{The Quadratic-Gaussian Rate-Distortion Function}

The rate-distortion function (RDF) of a stationary source with memory is given as a limit of normalized mutual information associated with vectors of source samples. For a real valued source $\left\{X_{n}\right\}=\ldots, X_{-2}, X_{-1}, X_{0}, X_{1}, X_{2}, \ldots$, and expected mean-squared distortion level $D$, the RDF can be written as, [2],

$$
R(D)=\lim _{n \rightarrow \infty} \frac{1}{n} \inf I\left(X_{1}, \ldots, X_{n} ; Y_{1}, \ldots, Y_{n}\right)
$$

\footnotetext{
${ }^{0}$ The work of the first two authors was partially supported by the Israel Science Foundation, grant ISF 1259/07
}

where the infimum is over all channels $\mathbf{X} \rightarrow \mathbf{Y}$ such that $\frac{1}{n}\|\mathbf{Y}-\mathbf{X}\|^{2} \leq D$. A channel which realizes this infimum is called an optimum test-channel. When the source is zero-mean Gaussian, the RDF takes an explicit form in the frequency domain in terms of the power-spectrum

$$
S\left(e^{j 2 \pi f}\right)=\sum_{k} R[k] e^{-j k 2 \pi f}, \quad-1 / 2<f<1 / 2,
$$

where $R[k]=E\left\{X_{n} X_{n+k}\right\}$ is the auto-correlation function of the source. The water filling solution, illustrated in Figure 2, gives a parametric formula for the Gaussian RDF in terms of a parameter $\theta[8],[2],[5]$ :

$$
\begin{aligned}
R(D) & =\int_{-1 / 2}^{1 / 2} \frac{1}{2} \log \left(\frac{S\left(e^{j 2 \pi f}\right)}{D\left(e^{j 2 \pi f}\right)}\right) d f \\
& =\int_{f: S\left(e^{j 2 \pi f}\right)>\theta} \frac{1}{2} \log \left(\frac{S\left(e^{j 2 \pi f}\right)}{\theta}\right) d f
\end{aligned}
$$

where the distortion spectrum is given by

$$
D\left(e^{j 2 \pi f}\right)= \begin{cases}\theta, & \text { if } S\left(e^{j 2 \pi f}\right)>\theta \\ S\left(e^{j 2 \pi f}\right), & \text { otherwise }\end{cases}
$$

and where we choose the water level $\theta$ so that the total distortion is $D$ :

$$
D=\int_{-1 / 2}^{1 / 2} D\left(e^{j 2 \pi f}\right) d f .
$$

In the special case of a memoryless (white) Gaussian source $\sim N\left(0, \sigma^{2}\right)$, the power-spectrum is flat $S\left(e^{j 2 \pi f}\right)=\sigma^{2}$, so $\theta=D$ and the RDF is simplified to

$$
\frac{1}{2} \log \left(\frac{\sigma^{2}}{D}\right), \quad 0<D \leq \sigma^{2} .
$$

The optimum test-channel can be written in this case in a backward additive-noise form: $X=Y+N$, with $N \sim$ $N(0, D)$, or in a forward linear additive-noise form:

$$
Y=\beta(\alpha X+N)
$$

with $\alpha=\beta=\sqrt{1-D / \sigma^{2}}$ and $N \sim N(0, D)$. In the general stationary case, the forward channel realization of the Gaussian RDF has several equivalent forms [8, Sec. 9.7], [2, Sec. 4.5]. The one which is more useful for our purpose replaces $\alpha$ and $\beta$ above by linear time-invariant filters, while keeping the noise $N$ as AWGN [18]:

$$
Y_{n}=h_{2, n} *\left(h_{1, n} * X_{n}+N_{n}\right)
$$

where $N_{n} \sim N(0, \theta)$ is AWGN with $\theta=\theta(D)=$ the water level, $*$ denotes convolution, and $h_{1, n}$ and $h_{2, n}$ are the impulse 


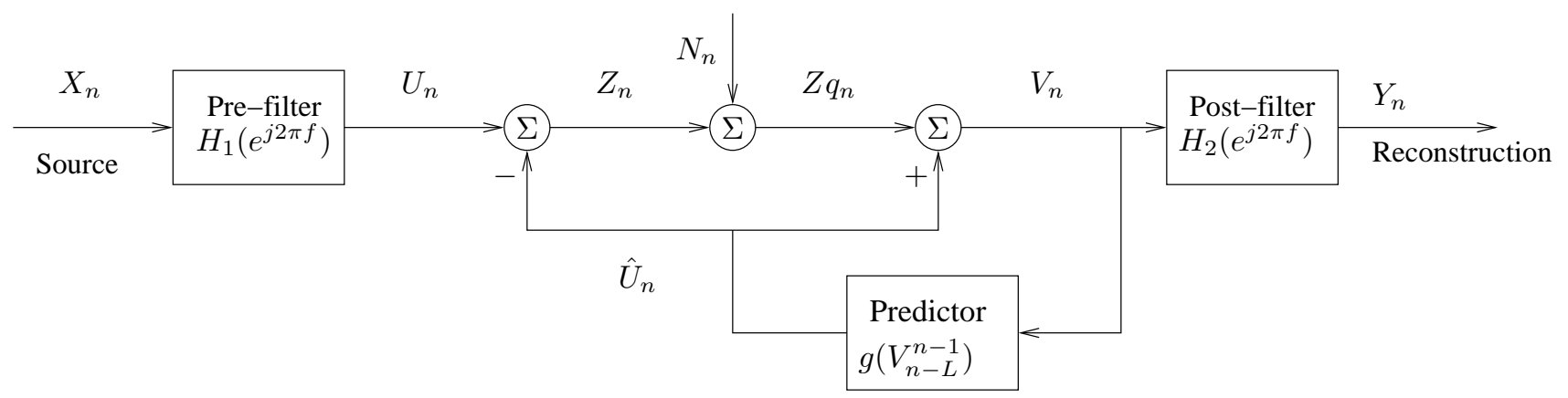

Fig. 1. Predictive Test Channel.

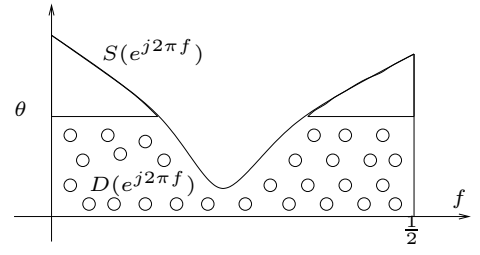

Fig. 2. The water filling solution.

responses of a suitable pre-filter and post-filter, respectively. See (13)-(18) in the next section.

If we take a discrete approximation of (1),

$$
\sum_{i} \frac{1}{2} \log \left(\frac{S\left(e^{j 2 \pi f_{i}}\right)}{D\left(e^{j 2 \pi f_{i}}\right)}\right),
$$

then each component has the memoryless form of (4). Hence, we can think of the frequency domain formula (1) as an encoding of parallel (independent) Gaussian sources, where source $i$ is a memoryless Gaussian source $X_{i} \sim N\left(0, S\left(e^{j 2 \pi f_{i}}\right)\right)$ encoded at distortion level $D\left(e^{j 2 \pi f_{i}}\right)$; see [5]. Indeed, practical frequency domain source coding schemes such as Transform Coding and Sub-band Coding [10] get close to the RDF of a stationary Gaussian source using an "array" of parallel scalar quantizers.

\section{Rate-Distortion and Prediction}

Our main result is a predictive channel realization for the quadratic-Gaussian RDF (1), which can be viewed as the time-domain counterpart of the frequency domain formulation above. The notions of entropy-power and Shannon lower bound (SLB) provide a simple relation between the Gaussian $\mathrm{RDF}$ and prediction, and motivate our result. Recall that the entropy-power is the variance of a white Gaussian process having the same entropy-rate as the source [5]; for a zero-mean Gaussian source with power-spectrum $S\left(e^{j 2 \pi f}\right)$, the entropypower is given by

$$
P_{e}(X)=\exp \left(\int_{-1 / 2}^{1 / 2} \log \left(S\left(e^{j 2 \pi f}\right)\right) d f\right) .
$$

In the context of Wiener's spectral-factorization theory, the entropy-power quantifies the MMSE in one-step linear prediction of a Gaussian source from its infinite past [2]:

$$
P_{e}(X)=\inf _{\left\{a_{i}\right\}} E\left(X_{n}-\sum_{i=1}^{\infty} a_{i} X_{n-i}\right)^{2} .
$$

The error process associated with the infinite-order optimum predictor,

$$
Z_{n}=X_{n}-\sum_{i=1}^{\infty} a_{i} X_{n-i},
$$

is called the innovation process. The orthogonality principle of MMSE estimation implies that the innovation process has zero mean and is white; in the Gaussian case un-correlation implies independence, so

$$
Z_{n} \sim \mathcal{N}\left(0, P_{e}(X)\right)
$$

is a memoryless process. See, e.g., [7].

¿From an information theoretic perspective, the entropypower plays a role in the SLB:

$$
R(D) \geq \frac{1}{2} \log \left(\frac{P_{e}(X)}{D}\right) .
$$

Equality in the SLB holds if the distortion level is smaller than or equal to the lowest value of the power spectrum: $D \leq$ $S_{\text {min }} \triangleq \min _{f} S\left(e^{j 2 \pi f}\right)$, in which case $D\left(e^{j 2 \pi f}\right)=\theta=D$ [2]. It follows that for distortion levels below $S_{\min }$ the RDF of a Gaussian source with memory is equal to the RDF of its memoryless innovation process $Z_{n}$ :

$$
R(D)=R_{Z}(D)=\frac{1}{2} \log \left(\frac{\sigma_{Z}^{2}}{D}\right), \quad D \leq S_{\min },
$$

where $\sigma_{Z}^{2}=P_{e}(X)$.

We shall see later in Section II how identity (12) translates into a predictive test-channel, which can realize the RDF not only for small but for all distortion levels. This test channel is motivated by the sequential structure of Differential Pulse Code Modulation (DPCM) [12], [10]. The goal of DPCM is to translate the encoding of dependent source samples into a series of independent encodings. The task of removing the time dependence is achieved by (linear) prediction: at each time instant the incoming source sample is predicted from previously encoded samples, the prediction error is encoded by a scalar quantizer and added to the predicted value to form the new reconstruction. See Figure 3 . 


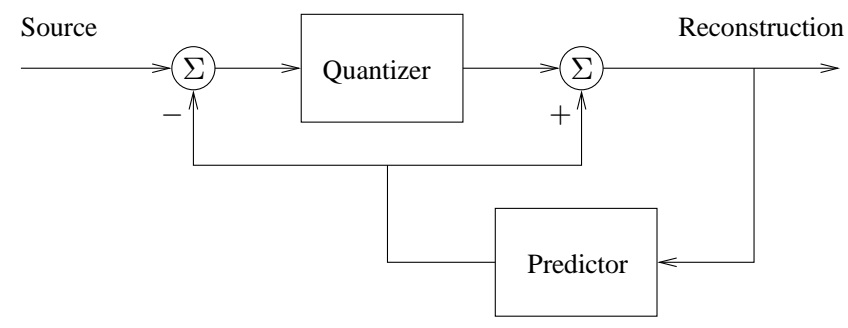

Fig. 3. DPCM Quantiztion Scheme.

A negative result along this direction was recently given by Kim and Berger [13]. They showed that the RDF of an auto-regressive (AR) Gaussian process cannot be achieved by directly encoding its innovation process. This can be viewed as open-loop prediction, because the innovation process is extracted from the clean source rather than from the quantized source [12], [9]. Here we give a positive result, showing that the RDF can be achieved if we embed the quantizer inside the prediction loop, i.e., by closed-loop prediction as done in DPCM. The RDF-achieving system consists of pre- and post-filters, and an AWGN channel embedded in a source prediction loop. As we show, the scalar (un-conditioned) mutual information over this inner AWGN channel is equal to the RDF.

After presenting and proving our main result in Sections II and III, respectively, we discuss its characteristics and operational implications. Section IV discusses the spectral features of the solution. Section V relates the solution to vector-quantized DPCM of parallel sources. Section VI shows an implementation by Entropy Coded Dithered Quantization (ECDQ), while extending the ECDQ rate formula [16] to the case of a system with feedback. Finally, in Section VII we relate prediction in source coding to prediction for channel equalization and to recent observations by Forney [7]. As in [7], our analysis is based on the properties of information measures; the only result we need from Wiener's estimation theory is the orthogonality principle.

\section{MAin Result}

Consider the system in Figure 1, which consists of three basic blocks: a pre-filter $H_{1}\left(e^{j 2 \pi f}\right)$, a noisy channel embedded in a closed loop, and a post-filter $H_{2}\left(e^{j 2 \pi f}\right)$, where $H\left(e^{j 2 \pi f}\right)$ denotes the frequency response of a filter with impulse response $h_{n}$,

$$
H\left(e^{j 2 \pi f}\right)=\sum_{n} h_{n} e^{-j n 2 \pi f}, \quad-1 / 2<f<1 / 2 .
$$

The system parameters are derived from the water-filling solution (1)-(2), and depend on the source spectrum $S\left(e^{j 2 \pi f}\right)$ and the distortion level $D$. The source samples $\left\{X_{n}\right\}$ are passed through a pre-filter, whose phase is arbitrary and its absolute squared frequency response is given by

$$
\left|H_{1}\left(e^{j 2 \pi f}\right)\right|^{2}=1-\frac{D\left(e^{j 2 \pi f}\right)}{S\left(e^{j 2 \pi f}\right)}
$$

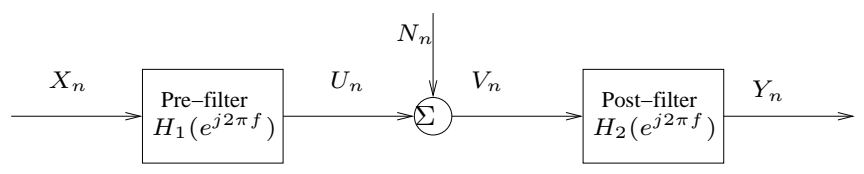

Fig. 4. Equivalent Channel.

where $\frac{0}{0}$ is taken as 1 . The pre-filter output, denoted $U_{n}$, is fed to the central block which generates a process $V_{n}$ according to the following recursion equations:

$$
\begin{aligned}
\hat{U}_{n} & =g\left(V_{n-1}, V_{n-2}, \ldots, V_{n-L}\right) \\
Z_{n} & =U_{n}-\hat{U}_{n} \\
Z q_{n} & =Z_{n}+N_{n} \\
V_{n} & =\hat{U}_{n}+Z q_{n}
\end{aligned}
$$

where $N_{n} \sim \mathcal{N}(0, \theta)$ is a zero-mean white Gaussian noise, independent of the input process $\left\{U_{n}\right\}$, whose variance is equal to the water level $\theta=\theta(D)$; and $g(\cdot)$ is some prediction function for the input $U_{n}$ given the $L$ past samples of the output process $\left(V_{n-1}, V_{n-2}, \ldots, V_{n-L}\right)$. 1 Finally, the postfilter frequency response is the complex conjugate of the frequency response of the pre-filter,

$$
H_{2}\left(e^{j 2 \pi f}\right)=H_{1}^{*}\left(e^{j 2 \pi f}\right) .
$$

Equivalently, the impulse response of the post-filter is the reflection of the impulse response of the pre-filter:

$$
h_{2, n}=h_{1,-n} .
$$

See a comment regarding causality in the end of the section.

The block from $U_{n}$ to $V_{n}$ is equivalent to the configuration of DPCM, [12], [10], with the DPCM quantizer replaced by the additive Gaussian noise channel $Z q_{n}=Z_{n}+N_{n}$. In particular, the recursion equations (14)-(17) imply that this block satisfies the well known "DPCM error identity", [12],

$$
V_{n}=U_{n}+\left(Z q_{n}-Z_{n}\right)=U_{n}+N_{n} .
$$

That is, the output $V_{n}$ is a noisy version of the input $U_{n}$ via the AWGN channel $V_{n}=U_{n}+N_{n}$. Thus, the system of Figure 1 is equivalent to the system depicted in Figure 4, which corresponds to the forward channel realization (5) of the quadratic-Gaussian RDF.

In DPCM the prediction function $g$ is linear:

$$
g\left(V_{n-1}, \ldots, V_{n-L}\right)=\sum_{i=1}^{L} a_{i} V_{n-i}
$$

where $a_{1}, \ldots, a_{L}$ are chosen to minimize the mean-squared prediction error:

$$
\sigma_{L}^{2}=\min _{a_{i}} E\left(U_{n}-\sum_{i=1}^{L} a_{i} V_{n-i}\right)^{2} .
$$

Because $V_{n}$ is the result of passing $U_{n}$ through an AWGN channel, we call that "noisy prediction". If $\left\{U_{n}\right\}$ and $\left\{V_{n}\right\}$ are jointly Gaussian, then the best predictor of any order is linear,

\footnotetext{
${ }^{1}$ No initial condition on $V_{n}$ is needed as we assume a two-sided input process $X_{n}$, and the system is stable.
} 
so $\sigma_{L}^{2}$ is also the MMSE in estimating $U_{n}$ from the vector $\left(V_{n-1}, \ldots, V_{n-L}\right)$. Clearly, this MMSE is non-increasing with the prediction order $L$, and as $L$ goes to infinity it converges to

$$
\sigma_{\infty}^{2}=\lim _{L \rightarrow \infty} \sigma_{L}^{2},
$$

the optimum infinite order prediction error in $U_{n}$ given the past

$$
V_{n}^{-} \triangleq\left\{V_{n-1}, V_{n-2}, \ldots\right\} .
$$

We shall see later in Section IV that $\sigma_{\infty}^{2}=P_{e}(V)-\theta$. We further elaborate on the relationship with DPCM in Section $\mathrm{V}$ We now state our main result.

Theorem 1: (Predictive test channel) For any stationary source with power spectrum $S\left(e^{j 2 \pi f}\right)$ and distortion level $D$, the system of Figure 1 with the pre-filter (13) and the postfilter (18), satisfies

$$
E\left(Y_{n}-X_{n}\right)^{2}=D
$$

Furthermore, if the source $X_{n}$ is Gaussian and $g=g\left(V_{n}^{-}\right)$ achieves the optimum infinite order prediction error $\sigma_{\infty}^{2}$, 23, then

$$
I\left(Z_{n} ; Z_{n}+N_{n}\right)=\frac{1}{2} \log \left(1+\frac{\sigma_{\infty}^{2}}{\theta}\right)=R(D),
$$

where the left hand side is the scalar mutual information over the channel (16).

The proof is given in Section III. The result above is in sharp contrast to the classical realization of the RDF (5), which involves mutual information rate over a test-channel with memory. In a sense, the core of the encoding process in the system of Figure 1 amounts to a memoryless AWGN testchannel (although, as we discuss in the sequel, the channel (16) is not quite memoryless nor additive). ¿From a practical perspective, this system provides a bridge between DPCM and rate-distortion theory for a general distortion level $D>0$.

Another interesting feature of the system is the relationship between the prediction error process $Z_{n}$ and the original process $X_{n}$. If $X_{n}$ is an auto-regressive (AR) process, then in the limit of small distortion $(D \rightarrow 0), Z_{n}$ is roughly its innovation process (9). Hence, unlike in open-loop prediction [13], encoding the innovations in a closed-loop system is optimal in the limit of high-resolution encoding. We shall return to this point, as well as discuss the case of general resolution, in Section IV

Finally, we note that while the central block of the system is sequential and hence causal, the pre- and post-filters are non-causal and therefore their realization in practice requires delay. Specifically, since by (19) $h_{2, n}=h_{1,-n}$, if one of the filters is causal then the other must be anti-causal. Often the filter's response is infinite, hence the required delay is infinite as well. Of course, one can approximate the desired spectrum (in $L_{2}$ sense and hence also in rate-distortion sense) to any degree using filters of sufficiently large but finite delay $\delta$, so the system distortion is actually measured between $Y_{n}$ and
$X_{n-\delta}$. In this sense, Theorem 1 holds in general in the limit as the system delay $\delta$ goes to infinity.

If we insist on a system with causal reconstruction $(\delta=$ $0)$, then we cannot realize the pre- and post-filters (13) and (18), and some loss in performance must be paid. Nevertheless, if the source spectrum is bounded from below by a positive constant, then it can be seen from (13) that in the limit of small distortion $(D \rightarrow 0)$ the filters can be omitted, i.e., $H_{1}=H_{2}=$ 1 for all $f$. Hence, a causal system (the central block in Figure 1) is asymptotically optimal at "high resolution" conditions. Furthermore, the redundancy of an AWGN channel above the $\mathrm{RDF}$ is at most 0.5 bit per source sample for any source and at any resolution; see, e.g., [16]. It thus follows from Lemma 1 below (which directly characterizes the information rate of the central block of Figure 1), that a causal system (the system of Figure 1 without the filters) loses at most 0.5 bit at any resolution.

These observations shed some light on the "cost of causality" in encoding stationary Gaussian sources [14]. It is an open question, though, whether a redundancy better than 0.5 bit can be guaranteed when using causal pre and post filters in the system of Figure 1.

\section{Proof of Main Result}

We start with Lemma 1 below, which shows an identity between the mutual information rate over the central block of Figure 1 and the scalar mutual information (26). This identity holds regardless of the pre- and post-filters, and only assumes optimum infinite order prediction in the feedback loop.

Let

$$
\bar{I}\left(\left\{U_{n}\right\} ;\left\{V_{n}\right\}\right)=\lim _{n \rightarrow \infty} \frac{1}{n} I\left(U_{1}, \ldots, U_{n} ; V_{1}, \ldots, V_{n}\right)
$$

denote mutual information-rate between jointly stationary sources $\left\{U_{n}\right\}$ and $\left\{V_{n}\right\}$, whenever the limit exists.

Lemma 1: For any stationary Gaussian process $\left\{U_{n}\right\}$ in Figure 1, if $\hat{U}_{n}$ is the optimum infinite order predictor of $U_{n}$ from $V_{n}^{-}$(so the variance of $Z_{n}$ is $\sigma_{\infty}^{2}$ as defined in (23)), then

$$
\bar{I}\left(\left\{U_{n}\right\} ;\left\{V_{n}\right\}\right)=I\left(Z_{n} ; Z_{n}+N_{n}\right)
$$

Proof: For any finite order predictor $g\left(V_{n-L}^{n-1}\right)$ we can write

$$
\begin{aligned}
I\left(\left\{U_{n}\right\} ; V_{i} \mid V_{i-L}^{i-1}\right) & =I\left(\left\{U_{n}\right\}, U_{i}-\hat{U}_{i}^{(L)} ; V_{i}-\hat{U}_{i}^{(L)} \mid V_{i-L}^{i-1}\right) \\
& =I\left(\left\{U_{n}\right\}, Z_{i}^{(L)} ; Z_{i}^{(L)}+N_{i} \mid V_{i-L}^{i-1}\right) \\
& =I\left(Z_{i}^{(L)} ; Z_{i}^{(L)}+N_{i} \mid V_{i-L}^{i-1}\right) \\
& =I\left(Z_{i}^{(L)} ; Z_{i}^{(L)}+N_{i}\right)
\end{aligned}
$$

where $\hat{U}_{i}^{(L)}=g\left(V_{i-L}^{i-1}\right)$ is the $L$-th order predictor output at time $i$, and $Z_{i}^{(L)}$ is the prediction error. The first equality above follows since manipulating the condition does not 
affect the conditional mutual information; the second equality follows from the definition of $Z_{i}^{(L)}$; (29) follows since $N_{i}$ is independent of $\left(\left\{U_{n}\right\}, V_{i}^{-}\right)$and therefore

$$
\left(Z_{i}^{(L)}+N_{i}\right) \longleftrightarrow\left(Z_{i}^{(L)}, V_{i-L}^{i-1}\right) \longleftrightarrow\left\{U_{n}\right\}
$$

form a Markov chain; and (30) follows from two facts: first, since $N_{i}$ is independent of $\left\{U_{i}\right\}$ and previous $N_{i}$ 's, it is also independent of the pair $\left(Z_{i}^{(L)}, V_{i-L}^{i-1}\right)$ by the recursive structure of the system; second, we assume optimum (MMSE) prediction, hence the orthogonality principle implies that the prediction error $Z_{i}^{(L)}$ is orthogonal to the measurements $V_{i-L}^{i-1}$, so by Gaussianity they are also independent, and hence by the two facts we have that $V_{i-L}^{i-1}$ is independent of the pair $\left(Z_{i}^{(L)}, N_{i}\right)$. Since by (22) the variance of the $L$-th order prediction error $Z_{i}^{(L)}$ is $\sigma_{L}^{2}$, while the variance of the noise $N_{i}$ is $\theta$, we thus obtained from (30)

$$
I\left(\left\{U_{n}\right\} ; V_{i} \mid V_{i-L}^{i-1}\right)=\frac{1}{2} \log \left(1+\frac{\sigma_{L}^{2}}{\theta}\right) .
$$

This implies in the limit as $L \rightarrow \infty$

$$
\begin{aligned}
I\left(\left\{U_{n}\right\} ; V_{i} \mid V_{i}^{-}\right) & =\frac{1}{2} \log \left(1+\frac{\sigma_{\infty}^{2}}{\theta}\right) \\
& =I\left(Z_{n} ; Z_{n}+N_{n}\right) .
\end{aligned}
$$

Note that by stationarity, $I\left(\left\{U_{n}\right\} ; V_{i} \mid V_{i}^{-}\right)$is independent of $i$. Thus,

$$
I\left(\left\{U_{n}\right\} ; V_{1}\right)+I\left(\left\{U_{n}\right\} ; V_{2} \mid V_{1}\right)+\ldots+I\left(\left\{U_{n}\right\} ; V_{i} \mid V_{1}^{i-1}\right)
$$

normalized by $1 / i$ converges as $i \rightarrow \infty$ to $I\left(\left\{U_{n}\right\} ; V_{i} \mid V_{i}^{-}\right)$. By the definition of mutual information rate (27) and by the chain rule for mutual information [5], this implies that the left hand side of (28) is equal to

$$
\bar{I}\left(\left\{U_{n}\right\} ;\left\{V_{n}\right\}\right)=I\left(\left\{U_{n}\right\} ; V_{i} \mid V_{i}^{-}\right) .
$$

Combining (33) and (34) the lemma is proved.

Theorem 1 is a simple consequence of Lemma 1 above and the forward channel realization of the RDF. As discussed in the previous section, the DPCM error identity (20) implies that the entire system of Figure 1 is equivalent to the system depicted in Figure 4, consisting of a pre-filter (13), an AWGN channel with noise variance $\theta$, and a post-filter (18). This is also the forward channel realization (5) of the RDF [8], [2], [18]. In particular, as simple spectral analysis shows, the power spectrum of the overall error process $Y_{n}-X_{n}$ is equal to the water filling distortion spectrum $D\left(e^{j 2 \pi f}\right)$ in (2). Hence, by (3) the total distortion is $D$, and (25) follows.

We turn to prove the second part of the theorem (equation (26) ). Since the system of Figure 4 is equivalent to the forward channel realization (5) of the RDF of $\left\{X_{n}\right\}$, we have

$$
\bar{I}\left(\left\{X_{n}\right\} ;\left\{Y_{n}\right\}\right)=R(D)
$$

where $\bar{I}$ denotes mutual information-rate (27). Since $\left\{U_{n}\right\}$ is a function of $\left\{X_{n}\right\}$, and since the post-filter $H_{2}$ is invertible within the pass-band of the pre-filter $H_{1}$, we also have

$$
\bar{I}\left(\left\{X_{n}\right\} ;\left\{Y_{n}\right\}\right)=\bar{I}\left(\left\{U_{n}\right\} ;\left\{V_{n}\right\}\right) .
$$

The theorem now follows by combining (36), (35) and Lemma 1

An alternative proof of Theorem 11 based only on spectral considerations, is given in the end of the next section.

\section{Properties of the Predictive Test-Channel}

The following observations shed light on the behavior of the test channel of Figure 1

Prediction in the high resolution regime. If the powerspectrum $S\left(e^{j 2 \pi f}\right)$ is everywhere positive (e.g., if $\left\{X_{n}\right\}$ can be represented as an AR process), then in the limit of small distortion $D \rightarrow 0$, the pre- and post-filters (13), 118) converge to all-pass filters, and the power spectrum of $U_{n}$ becomes the power spectrum of the source $X_{n}$. Furthermore, noisy prediction of $U_{n}$ (from the "noisy past" $V_{n}^{-}$, where $V_{n}=$ $U_{n}+N_{n}$ ) becomes equivalent to clean prediction of $U_{n}$ from its own past $U_{n}^{-}$. Hence, in this limit the prediction error $Z_{n}$ is equivalent to the innovation process of $X_{n}$ (9). In particular, $Z_{n}$ is an i.i.d. process whose variance is $P_{e}(X)=$ the entropypower of the source (7).

Prediction in the general case. Interestingly, for general distortion $D>0$, the prediction error $Z_{n}$ is not white, as the noisiness of the past does not allow the predictor $g$ to remove all the source memory. Nevertheless, the noisy version of the prediction error $Z q_{n}=Z_{n}+N_{n}$ is white for every $D>0$, because it amounts to predicting $V_{n}$ from its own infinite past: since $N_{n}$ has zero-mean and is white (and therefore independent of the past), $\hat{U}_{n}$ that minimizes the prediction error of $U_{n}$ is also the optimal predictor for $V_{n}=U_{n}+N_{n}$. In particular, in view of (8) and (10), we have

$$
Z q_{n} \sim \mathcal{N}\left(0, P_{e}(V)\right)
$$

where $P_{e}(V)$ is the entropy-power of the process $V_{n}$. And since $Z q_{n}$ is the independent sum of $Z_{n}$ and $N_{n}$, we also have the relation

$$
P_{e}(V)=\sigma_{\infty}^{2}+\theta
$$

where $\sigma_{\infty}^{2}$ is the variance of $Z_{n}(23)$ and $\theta$ is the variance of $N_{n}$.

Sequential Additivity. The whiteness of $Z q_{n}$ might seem at first a contradiction, because $Z q_{n}$ is the sum of a non-white process, $Z_{n}$, and a white process $N_{n}$; nevertheless, $\left\{Z_{n}\right\}$ and $\left\{N_{n}\right\}$ are not independent, because $Z_{n}$ depends on past values of $N_{n}$ through the feedback loop and the past of $V_{n}$. Thus, the channel $Z q_{n}=Z_{n}+N_{n}$ is not quite additive but "sequentially additive": each new noise sample is independent of the present and the past but not necessarily of the future. In particular, this channel satisfies:

$I\left(Z_{n} ; Z_{n}+N_{n} \mid Z_{1}+N_{1}, \ldots, Z_{n-1}+N_{n-1}\right)=I\left(Z_{n} ; Z_{n}+N_{n}\right)$,

so by the chain rule for mutual information

$$
\bar{I}\left(\left\{Z_{n}\right\} ;\left\{Z_{n}+N_{n}\right\}\right)>I\left(Z_{n} ; Z_{n}+N_{n}\right) .
$$

Later in Section $\nabla \mathrm{D}$ we rewrite $(38)$ in terms of directed mutual information. 
The channel when the SLB is tight. As long as $D$ is smaller than the lowest point of the source power spectrum $S_{m i n}$, we have $D\left(e^{j 2 \pi f}\right)=\theta=D$ in (1), and the quadraticGaussian RDF coincides with the SLB (11). In this case, the following properties hold for the predictive test channel:

- The power spectra of $U_{n}$ and $Y_{n}$ are the same and are equal to $S\left(e^{j 2 \pi f}\right)-D$.

- The power spectrum of $V_{n}$ is equal to the power spectrum of the source $S\left(e^{j 2 \pi f}\right)$.

- The variance of $Z q_{n}$ is equal to the entropy-power of $V_{n}$ by (37), which is equal to $P_{e}(X)$.

- As a consequence we have

$$
\begin{aligned}
I\left(Z_{n} ; Z_{n}+N_{n}\right) & =h\left(Z q_{n}\right)-h\left(N_{n}\right) \\
& =h\left(\mathcal{N}\left(0, P_{e}(V)\right)\right)-h(\mathcal{N}(0, D)) \\
& =\frac{1}{2} \log \left(\frac{P_{e}(X)}{D}\right)
\end{aligned}
$$

which is indeed the SLB (11).

As discussed in the Introduction, the SLB is also the RDF of the innovation process (12), i.e., the conditional RDF of the source $X_{n}$ given its infinite clean past $X_{n}^{-}$.

An alternative derivation of Theorem 1 in the spectral domain. For a general $D$, we can use (37) and the equivalent channel of Figure 4 to re-derive the scalar mutual information RDF identity (26). Note that for any $D$ the power spectrum of $U_{n}$ and $Y_{n}$ is equal to $\max \left\{0, S\left(e^{j 2 \pi f}\right)-\theta\right\}$, where $\theta=\theta(D)$ is the water-level. Thus the power spectrum of $V_{n}=U_{n}+N_{n}$ is given by $\max \left\{\theta, S\left(e^{j 2 \pi f}\right)\right\}$. Since as discussed above the variance of $Z q_{n}=Z_{n}+N_{n}$ is given by the entropy power of the process $V_{n}$, we have

$$
\begin{aligned}
I\left(Z_{n} ; Z_{n}+N_{n}\right) & =\frac{1}{2} \log \left(\frac{P_{e}\left(\max \left\{\theta, S\left(e^{j 2 \pi f}\right)\right\}\right)}{\theta}\right) \\
& =R(D)
\end{aligned}
$$

where $P_{e}(\cdot)$ as a function of the spectrum is given in (7), and the second equality follows from (1).

\section{VeCTOR-QuAnTIZED DPCM AND D*PCM}

As mentioned earlier, the structure of the central block of the channel of Figure 1 is of a DPCM encoder, with the scalar quantizer replaced by the AWGN channel $Z q_{n}=$ $Z_{n}+N_{n}$. However, if we wish to implement the additive noise by a quantizer whose rate is the mutual information $I\left(Z_{n} ; Z_{n}+N_{n}\right)$, we must use vector quantization (VQ). Indeed, while scalar quantization noise is approximately uniform over intervals, good high dimensional lattices generate near Gaussian quantization noise [17]. Yet, how can we combine VQ and DPCM without violating the sequential nature of the system? In particular, the quantized sample $Z q_{n}$ must be available to generate $V_{n}$, before the system can predict $U_{n+1}$ and generate $Z_{n+1}$.

One way we can achieve the VQ gain and still retain the sequential structure of the system is by adding a "spatial" dimension, i.e., by jointly encoding a large number of parallel

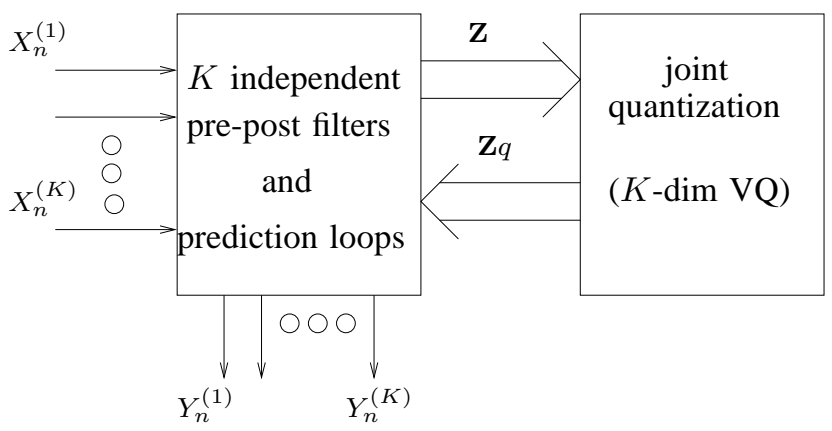

Fig. 5. DPCM of parallel sources.

sources, as happens, e.g., in video coding. Figure 5 shows DPCM encoding of $K$ parallel sources. The spectral shaping and prediction are done in the time domain for each source separately. Then, the resulting vector of $K$ prediction errors is quantized jointly at each time instant by a vector quantizer. The desired properties of additive quantization error, and rate which is equal to $K$ times the mutual information $I\left(Z_{n} ; Z_{n}+\right.$ $N_{n}$ ), can be approached in the limit of large $K$ by a suitable choice of the quantizer. In the next section we discuss one way to do that using lattice ECDQ.

What if we have only one source instead of $K$ parallel sources? If the source has decaying memory, we can still approximate the parallel source coding approach above, at the cost of large delay, by using interleaving. We divide the (pre-filtered) source into $K$ long blocks, which are separately predicted and then interleaved and jointly quantized as if they were parallel sources. See Figure ??. This is analogous to the method used in [11] for combining coding-decoding and decision-feedback equalization (DFE).

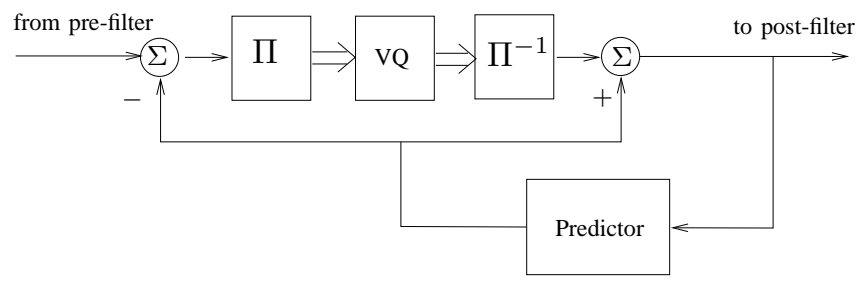

Fig. 6. VQ-DPCM for a single source using interleaving. ( $\Pi$ and $\Pi^{-1}$ denote interleaving and de-interleaving, respectively.)

If we do not use any of the above, but restrict ourselves to scalar quantization $(K=1)$, then we have a pre/post filtered DPCM scheme. By combining Theorem 1 with known bounds on the performance of (memoryless) entropy-constrained scalar quantizers (e.g., [18]), we have

$$
H\left(Q^{o p t}\left(Z_{n}\right)\right) \leq R(D)+\frac{1}{2} \log \left(\frac{2 \pi e}{12}\right)
$$

where $1 / 2 \log (2 \pi e / 12) \approx 0.254$ bit. See Remark 3 in the next section regarding scalar/lattice ECDQ. Hence, Theorem 1 implies that in principle, a pre/post filtered DPCM scheme is optimal, up to the loss of the VQ gain, at all distortion levels and not only at the high resolution regime.

A different approach to combine VQ and prediction is first to extract the innovation process and then to quantize 


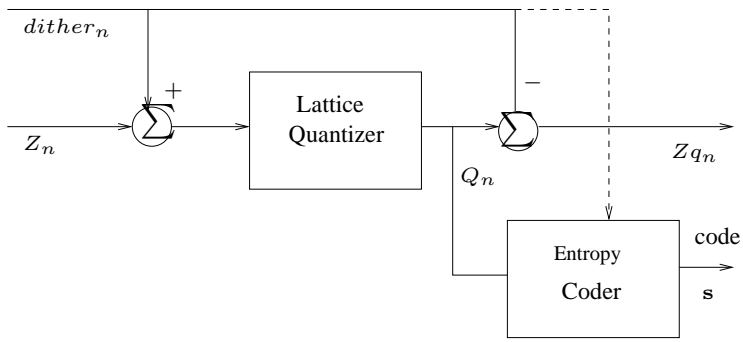

Fig. 7. ECDQ Structure.

it. It is interesting to mention that this method of "open loop" prediction, which we mentioned earlier regarding the model of [13], is known in the quantization literature as $\mathrm{D}^{*} \mathrm{PCM}[12]$. The best pre-filter for $\mathrm{D}^{*} \mathrm{PCM}$ under a high resolution assumption turns out to be the "half-whitening filter": $\left|H_{1}\left(e^{j 2 \pi f}\right)\right|^{2}=1 / \sqrt{S\left(e^{j 2 \pi f}\right)}$, with the post filter $H_{2}\left(e^{j 2 \pi f}\right)$ being its inverse. But even with this optimum filter, $\mathrm{D}^{*} \mathrm{PCM}$ is inferior to DPCM: The optimal distortion gain of $\mathrm{D}^{*} \mathrm{PCM}$ over a non-predictive scheme is

$$
G_{\mathrm{D}^{*} \mathrm{PCM}}=\frac{\sigma_{X}^{2}}{\left(\int_{-1 / 2}^{1 / 2} \sqrt{S_{X}\left(e^{j 2 \pi f}\right)} d f\right)^{2}}
$$

(strictly greater than one for non-white spectra by the CauchySchwartz inequality). Comparing to the optimum prediction gain obtained by the DPCM scheme:

$$
G_{\mathrm{DPCM}}=\frac{\sigma_{X}^{2}}{P_{e}(X)}
$$

we have:

$$
\frac{G_{\mathrm{DPCM}}}{G_{\mathrm{D}^{*} \mathrm{PCM}}}=\frac{\left(\int_{-1 / 2}^{1 / 2} \sqrt{S_{X}\left(e^{j 2 \pi f}\right)} d f\right)^{2}}{P_{e}(X)}=\left(\frac{\sigma_{\tilde{U}}^{2}}{P_{e}(\tilde{U})}\right)^{2},
$$

where $\tilde{U}_{n}$ is the pre-filter output in the $\mathrm{D}^{*} \mathrm{PCM}$ scheme. This ratio is strictly greater than one for non-white spectra.

\section{ECDQ in A CLOSEd Loop System}

Subtractive dithering of a uniform/lattice quantizer is a common approach to make the quantization noise additive. As shown in [16], the conditional entropy of the dithered lattice quantizer (given the dither) is equal to the mutual information in an additive noise channel, where the noise is uniform over the lattice cell. Furthermore, for "good" high dimensional lattices, the noise becomes closer to a white Gaussian process [17]. Thus, ECDQ (entropy-coded dithered quantization) provides a natural way to realize the inner AWGN channel block of the predictive test-channel.

One difficulty, however, we observe in this section is that the results developed in [16] do not apply to the case where the ECDQ input depends on previous EDCQ outputs and the entropy coding is conditioned on the past. This situation indeed happens in predictive coding, when ECDQ is embedded within a feedback loop. As we shall see, the right measure in this case is the directed information.
An ECDQ operating on the source $Z_{n}$ is depicted in Figure 7 A dither sequence $D_{n}$, independent of the input sequence $Z_{n}$, is added before the quantization and subtracted after. If the quantizer has a lattice structure of dimension $K \geq 1$, then we assume that the sequence length is

$$
L=M K
$$

for some integer $M$, so the quantizer is activated $M$ times. In this section, we use bold notation for $K$-blocks corresponding to a single quantizer operation. At each quantizer operation instant $m$, a dither vector $\mathbf{D}_{m}$ is independently and uniformly distributed over the basic lattice cell. The lattice points at the quantizer output $\mathbf{Q}_{m}, m=1, \ldots, M$ are fed into an entropy coder which is allowed to jointly encode the sequence, and has knowledge of the dither as well, thus for an input sequence of length $L$ it achieves an average rate of:

$$
R_{E C D Q} \triangleq \frac{1}{L} H\left(\mathbf{Q}_{1}^{M} \mid \mathbf{D}_{1}^{M}\right)
$$

bit per source sample. The entropy coder produces a sequence $\mathbf{s}$ of $\left\lceil L R_{E C D Q}\right\rceil$ bits, from which the decoder can recover $\mathbf{Q}_{1}, \ldots \mathbf{Q}_{M}$, and then subtract the dither to obtain the reconstruction sequence $Z q_{n}=Q_{n}-D_{n}, n=1, \ldots L$. The reconstruction error sequence

$$
N_{n}=Z q_{n}-Z_{n},
$$

called in the sequel the "ECDQ noise", has $K$-blocks which are uniformly distributed over the mirror image of the basic lattice cell and are mutually i.i.d. [16]. It is further stated in [16, Thm.1] that the input and the noise sequences, $\mathbf{Z}=Z_{1}^{L}$ and $\mathbf{N}=N_{1}^{L}$, are statistically independent, and that the ECDQ rate is equal to the mutual information over an additive noise channel with the input $\mathbf{Z}$ and the noise $\mathbf{N}$ :

$$
\begin{aligned}
R_{E C D Q} & =\frac{1}{L} I(\mathbf{Z} ; \mathbf{Z q}) \\
& =\frac{1}{L} I(\mathbf{Z} ; \mathbf{Z}+\mathbf{N}) .
\end{aligned}
$$

However, the derivation of [16, Thm. 1] makes the implicit assumption that the quantizer is used without feedback, that is, the current input is conditionally independent of past outputs given the past inputs. (In other words, the dependence on the past, if exists, is only due to memory in the source.) When there is feedback, this condition does not necessarily hold, which implies that (even with the dither) the sequences $\mathbf{Z}$ and $\mathbf{N}$ are possibly dependent. Specifically, since feedback is causal, the input $Z_{n}$ can depend on past values of the ECDQ noise $N_{n}$, so their joint distribution in general has the form:

$$
f\left(Z_{1}^{L}, N_{1}^{L}\right)=\prod_{m=1}^{M} f\left(\mathbf{N}_{m}\right) f\left(\mathbf{Z}_{m} \mid \mathbf{N}_{1}^{m-1}\right)
$$

where

$$
\mathbf{Z}_{m}=Z_{(m-1) K+1}^{m K}
$$

denotes the $m$ th $K$-block, and similarly for $\mathbf{N}_{m}$. In this case, the mutual information rate of (41) over-estimates the true rate of the ECDQ.

Massey shows in [15] that for DMCs with feedback, traditional mutual information is not a suitable measure, and should 
be replaced by directed information. The directed information between the sequences $\mathbf{Z}$ and $\mathbf{Z} \mathbf{q}=Z q_{1}^{L}$ is defined as

$$
\begin{aligned}
I(\mathbf{Z} \rightarrow \mathbf{Z q}) & \triangleq \sum_{n=1}^{L} I\left(Z_{1}^{n} ; Z q_{n} \mid Z q_{1}^{n-1}\right) \\
& =\sum_{n=1}^{L} I\left(Z_{n} ; Z q_{n} \mid Z q_{1}^{n-1}\right)
\end{aligned}
$$

where the second equality holds whenever the channel from $Z_{n}$ to $Z q_{n}$ is memoryless, as in our case. In contrast, the mutual information between $\mathbf{Z}$ and $\mathbf{Z} \mathbf{q}$ is given by,

$$
I(\mathbf{Z} ; \mathbf{Z q})=\sum_{n=1}^{L} I\left(Z_{1}^{L} ; Z q_{n} \mid Z q_{1}^{n-1}\right)
$$

which by the chain rule for mutual information is in general higher. For our purposes, we will define the $K$-block directed information:

$$
I_{K}(\mathbf{Z} \rightarrow \mathbf{Z} \mathbf{q}) \triangleq \sum_{m=1}^{M} I\left(\mathbf{Z}_{1}^{m} ; \mathbf{Z q}_{m} \mid \mathbf{Z} q_{1}^{m-1}\right)
$$

The following result, proven in Appendix A, extends Massey's observation to ECDQ with feedback, and generalizes the result of [16, Thm. 1]:

Theorem 2: (ECDQ Rate with Feedback) The ECDQ system with causal feedback defined by (42) satisfies:

$$
R_{E C D Q}=\frac{1}{L} I_{K}(\mathbf{Z} \rightarrow \mathbf{Z q})=\frac{1}{L} I_{K}(\mathbf{Z} \rightarrow \mathbf{Z}+\mathbf{N})
$$

\section{Remarks:}

1. When there is no feedback, the past and future input blocks $\left(\mathbf{Z}_{1}^{m-1}, \mathbf{Z}_{m+1}^{M}\right)$ are conditionally independent of the current output block $\mathbf{Z} q_{m}$ given the current input block $\mathbf{Z}_{m}$, implying by the chain rule that (43) coincides with (44), and Theorem 2 reduces to [16, Thm. 1].

2. Even for scalar quantization $(K=1)$, the ECDQ rate (40) refers to joint entropy coding of the whole input vector. This does not contradict the sequential nature of the system since entropy coding can be implemented causally. Indeed, it follows from the chain rule for entropy that it is enough to encode the instantaneous quantizer output $\mathbf{Q}_{m}$ conditioned on past quantizer outputs $\mathbf{Q}_{1}^{m-1}$ and on past and present dither samples $\mathbf{D}_{1}^{m}$, in order to achieve the joint entropy of the quantizer in (40).

3. If we don't condition the entropy coding on the past, then we have

$$
\begin{aligned}
R_{E C D Q} & =I\left(Z_{n} ; Z_{n}+N_{n}^{(\text {uniform })}\right) \\
& \leq I\left(Z_{n} ; Z_{n}+N_{n}^{(\text {gauss })}\right)+\frac{1}{2} \log \left(\frac{2 \pi e}{12}\right) \\
& =R(D)+\frac{1}{2} \log \left(\frac{2 \pi e}{12}\right)
\end{aligned}
$$

where $N_{n}^{\text {(uniform) }}$, the scalar quantization noise, is uniformly distributed over the interval $(-\sqrt{12 D},+\sqrt{12 D})$, and where
(49) follows from Theorem 11. This implies (39) in the previous section.

4. We can embed a $K$-dimensional lattice ECDQ for $K>1$ in the predictive test channel of Figure 11 instead of the additive noise channel, using the Vector-DPCM (VDPCM) configuration discussed in the previous section. For good lattices, when the quantizer dimension $K \rightarrow \infty$, the noise process $\mathbf{N}$ in the rate expressions (41) and (46) becomes white Gaussian, and the scheme achieves the rate-distortion function. Indeed, combining Theorems 1 and 2, we see that the average rate per sample of such VDPCM with ECDQ satisfies:

$$
R_{V D P C M-E C D Q}=I\left(Z_{n} ; Z_{n}+N_{n}\right)=R(D) .
$$

This implies, in particular, that the entropy coder does not need to be conditioned on the past at all, as the predictor handles all the memory. However, when the quantization noise is not Gaussian, or the predictor is not optimal, the entropy coder can use the residual time-dependence after prediction to further reduce the coding rate. The resulting rate of the ECDQ would be the average directed information between the source and its reconstruction as stated in Theorem 2 .

\section{A DuAl Relationship With DeCision-FEEDBACK EQUALIZATION}

In this section we make an analogy between the predictive form of the Gaussian RDF and the "information-optimality" of decision-feedback equalization (DFE) for colored Gaussian channels. As we shall see, a symmetric equivalent form of this channel coding problem, including a water-pouring transmission filter, an MMSE receive filter and a noise prediction feedback loop, exhibits a striking resemblance to the pre/postfiltered predictive test-channel of Figure 1

We consider the (real-valued) discrete-time time-invariant linear Gaussian channel,

$$
R_{n}=c_{n} * X_{n}+Z_{n},
$$

where the transmitted signal $S_{n}$ is subject to a power constraint $E\left[S_{n}^{2}\right] \leq P$, the channel dispersion is modeled by a linear time-invariant filter $c_{n}$, and where the channel noise $Z_{n}$ is (possibly colored) Gaussian noise.

Let $U_{n}$ represent the data stream which we model as an i.i.d. zero-mean Gaussian random process with variance $\sigma_{U}^{2}$. Further, let $h_{1, n}$ be a spectral shaping filter, satisfying

$$
\sigma_{U}^{2} \int_{-1 / 2}^{1 / 2}\left|H_{1}\left(e^{j 2 \pi f}\right)\right|^{2} d f \leq P
$$

so the channel input $X_{n}=h_{1, n} * U_{n}$ indeed satisfies the power constraint. For the moment, we make no further assumption on $h_{n}$.

The channel (52) has inter-symbol interference (ISI) due to the channel filter $c_{n}$, as well as colored Gaussian noise. Let us assume that the channel frequency response is non-zero everywhere, and pass the received signal $R_{n}$ through a zeroforcing (ZF) linear equalizer $\frac{1}{C(z)}$, resulting in $Y_{n}$. We thus 


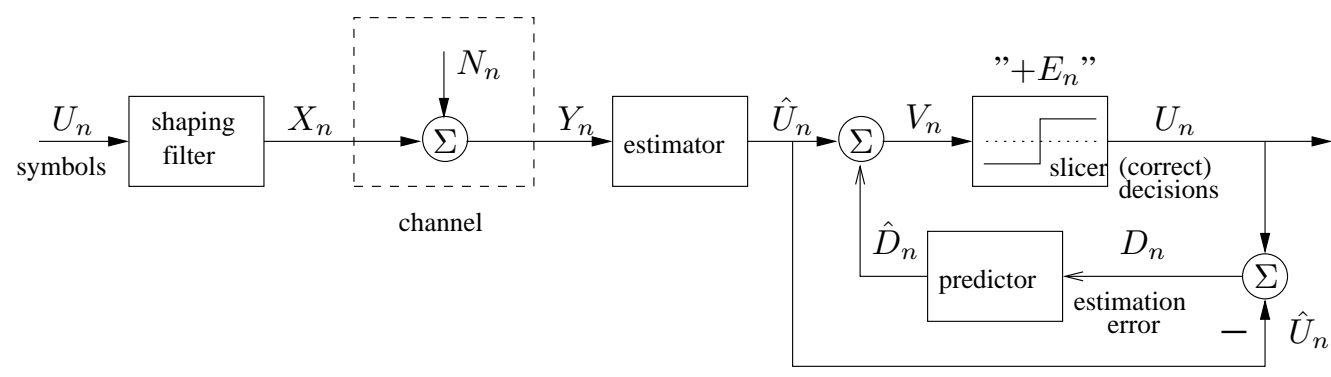

Fig. 8. MMSE-DFE Scheme in Predictive Form

arrive at an equivalent ISI-free channel,

$$
Y_{n}=X_{n}+N_{n}
$$

where the power spectrum of $N_{n}$ is

$$
S_{N}\left(e^{j 2 \pi f}\right)=\frac{S_{Z}\left(e^{j 2 \pi f}\right)}{\left|C\left(e^{j 2 \pi f}\right)\right|^{2}} .
$$

The mutual information rate (normalized per symbol) 27 between the input and output of the channel (52) is

$$
\bar{I}\left(\left\{X_{n}\right\},\left\{Y_{n}\right\}\right)=\int_{-1 / 2}^{1 / 2} \frac{1}{2} \log \left(1+\frac{S_{X}\left(e^{j 2 \pi f}\right)}{S_{N}\left(e^{j 2 \pi f}\right)}\right) d f .
$$

We note that if the spectral shaping filter $h_{n}$ satisfies the optimum "water-filling" power allocation condition, [5], then (53) will equal the channel capacity.

Similarly to the observations made in Section \with respect to the RDF, we note (as reflected in (53)) that capacity may be achieved by parallel AWGN coding over narrow frequency bands (as done in practice in Discrete Multitone (DMT)/Orthogonal Frequency-Division Multiplexing (OFDM) systems). An alternative approach, based on time-domain prediction rather than the Fourier transform, is offered by the canonical MMSE - feed forward equalizer - decision feedback equalizer (FFE-DFE) structure used in single-carrier transmission. It is well known that this scheme, coupled with AWGN coding, can achieve the capacity of linear Gaussian channels. This has been shown using different approaches by numerous authors; see [11], [4], [1], [7] and references therein. Our exposition closely follows that of Forney [7]. We now recount this result, based on linear prediction of the error sequence; see the system in Figure 8 and its equivalent channel in Figure 9. In the communication literature, this structure is referred to as "noise prediction". It can be recast into the more familiar FFE-DFE form by absorbing a part of the predictor into the estimator filter, forming the usual FFE.

As a first step, let $\hat{U}_{n}$ be the optimal MMSE estimator of $U_{n}$ from the equivalent channel output sequence $\left\{Y_{n}\right\}$ of (52). Since $\left\{U_{n}\right\}$ and $\left\{Y_{n}\right\}$ are jointly Gaussian and stationary this estimator is linear and time invariant. Note that the combination of the $\mathrm{ZF}$ equalizer $\frac{1}{C(z)}$ at the receiver frontend and the estimator above is equivalent to direct MMSE estimation of $U_{n}$ from the original channel output $R_{n}$ (50). Denote the estimation error, which is composed in general of ISI and Gaussian noise, by $D_{n}$. Then

$$
U_{n}=\hat{U}_{n}+D_{n}
$$

where $\left\{D_{n}\right\}$ is statistically independent of $\left\{\hat{U}_{n}\right\}$ due to the orthogonality principle and Gaussianity.

Assuming the decoder has access to past symbols $U_{n}^{-}=$ $U_{n-1}, U_{n-2}, \ldots$ (see in the sequel), the decoder knows also the past estimation errors $D_{n}^{-}=D_{n-1}, D_{n-2}, \ldots$ and may form an optimal linear predictor, $\hat{D}_{n}$, of the current estimation error $D_{n}$, which may then be added to $\hat{U}_{n}$ to form $V_{n}$. The prediction error $E_{n}=D_{n}-\hat{D}_{n}$ has variance $P_{e}(D)$, the entropy power of $D_{n}$. It follows that

$$
\begin{aligned}
U_{n} & =\hat{U}_{n}+D_{n} \\
& =V_{n}-\hat{D}_{n}+D_{n} \\
& =V_{n}+E_{n},
\end{aligned}
$$

and therefore

$$
E\left\{U_{n}-V_{n}\right\}^{2}=\sigma_{E}^{2}=E\left\{D_{n}-\hat{D}_{n}\right\}^{2}=P_{e}(D) .
$$

The channel (55), which describes the input/output relation of the slicer in Figure 8, is often referred to as the backward channel. Furthermore, since $U_{n}$ and $E_{n}$ are i.i.d Gaussian and since by the orthogonality principle $E_{n}$ is independent of present and past values of $V_{n}$ (but dependent of future values through the feedback loop), it is a "sequentially additive" AWGN channel. See Figure 10 for a geometric view of these properties. Notice the strong resemblance with the channel (16), $Z q_{n}=Z_{n}+N_{n}$, in the predictive test-channel of the RDF: in both channels the output and the noise are i.i.d. and Gaussian, but the input has memory and it depends on past outputs via the feedback loop.

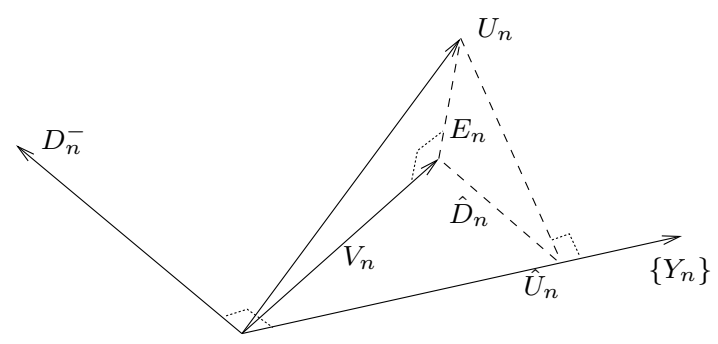

Fig. 10. Geometric View of the Estimation Process

We have therefore derived the following.

Theorem 3: (Information Optimality of Noise Prediction) For stationary Gaussian processes $U_{n}$ and $N_{n}$, and if $H_{2}\left(e^{j 2 \pi f}\right)$ is chosen to be the optimal estimation filter of $U_{n}$ 


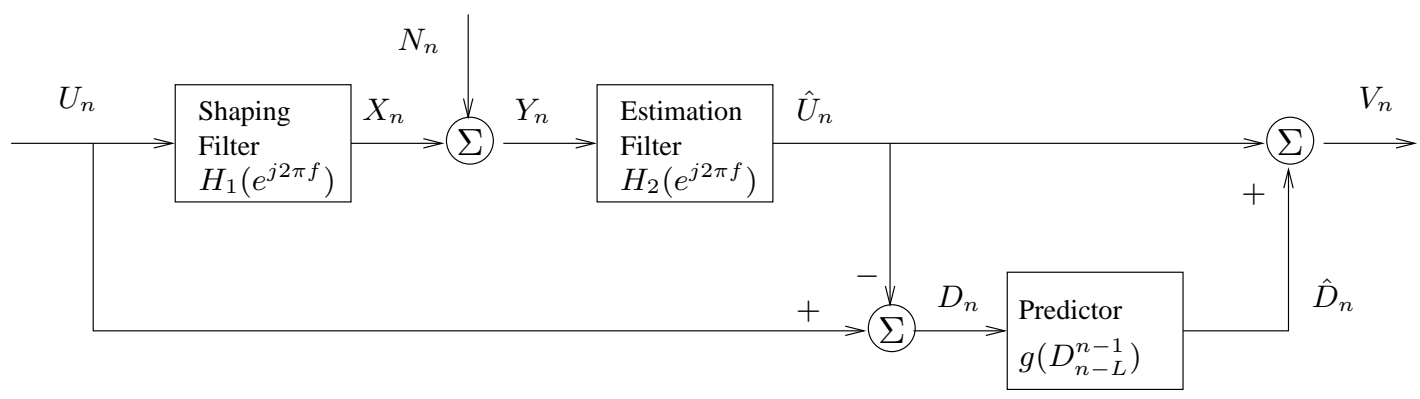

Fig. 9. Noise-Prediction Equivalent Channel

from $Y_{n}$ and the predictor $g(\cdot)$ is chosen to be the optimal prediction filter of $D_{n}$ (with $L \rightarrow \infty$ ), then the mutual information-rate (53) of the channel from $X_{n}$ to $Y_{n}$ (or from $U_{n}$ to $\left.Y_{n}\right)$ is equal to the scalar mutual information

$$
I\left(V_{n} ; V_{n}+E_{n}\right)
$$

of the channel (55). Furthermore, if $H_{1}\left(e^{j 2 \pi f}\right)$ is chosen such that $S_{X}\left(e^{j 2 \pi f}\right)$ equals the water-filling spectrum of the channel input, then this mutual information equals the channel capacity.

Proof: Let $U_{n}^{-}=\left\{U_{n-1}, U_{n-2}, \ldots\right\}$ and $D_{n}^{-}=$ $\left\{D_{n-1}, D_{n-2}, \ldots\right\}$. Using the chain rule of mutual information we have

$$
\begin{aligned}
\bar{I}\left(\left\{U_{n}\right\},\left\{Y_{n}\right\}\right) & =\bar{h}\left(\left\{U_{n}\right\}\right)-\bar{h}\left(\left\{U_{n}\right\} \mid\left\{Y_{n}\right\}\right) \\
& =\bar{h}\left(\left\{U_{n}\right\}\right)-h\left(U_{n} \mid\left\{Y_{n}\right\}, U_{n}^{-}\right) \\
& =\bar{h}\left(\left\{U_{n}\right\}\right)-h\left(U_{n}-\hat{U}_{n} \mid\left\{Y_{n}\right\}, U_{n}^{-}\right) \\
& =\bar{h}\left(\left\{U_{n}\right\}\right)-h\left(D_{n} \mid\left\{Y_{n}\right\}, U_{n}^{-}\right) \\
& =\bar{h}\left(\left\{U_{n}\right\}\right)-h\left(D_{n} \mid\left\{Y_{n}\right\}, D_{n}^{-}\right) \\
& =\bar{h}\left(\left\{U_{n}\right\}\right)-h\left(D_{n}-\hat{D}_{n} \mid\left\{Y_{n}\right\}, D_{n}^{-}\right) \\
& =\bar{h}\left(\left\{U_{n}\right\}\right)-h\left(E_{n} \mid\left\{Y_{n}\right\}, D_{n}^{-}\right) \\
& =\bar{h}\left(\left\{U_{n}\right\}\right)-h\left(E_{n}\right) \\
& =I\left(V_{n} ; V_{n}+E_{n}\right),
\end{aligned}
$$

where $\bar{h}(\cdot)$ denotes differential entropy rate, and where 57 follows from successive application of the orthogonality principle [7], since we assumed optimum estimation and prediction filters, which are MMSE estimators in the Gaussian setting.

In view of (53) and (56), and since $\bar{I}\left(\left\{U_{n}\right\},\left\{Y_{n}\right\}\right)=$ $\bar{I}\left(\left\{X_{n}\right\},\left\{Y_{n}\right\}\right)$, Theorem 3 can be re-written as

$$
\int_{-1 / 2}^{1 / 2} \frac{1}{2} \log \left(1+\frac{S_{X}\left(e^{j 2 \pi f}\right)}{S_{N}\left(e^{j 2 \pi f}\right)}\right) d f=\frac{1}{2} \log \left(\frac{\sigma_{U}^{2}}{\sigma_{E}^{2}}\right)
$$

from which we obtain the following well known formula for the "SNR at the slicer" for infinite order FFE-DFE, [4], [1],

$$
\frac{\sigma_{U}^{2}}{\sigma_{E}^{2}}=\exp \left(\int_{-1 / 2}^{1 / 2} \log \left(1+\frac{S_{X}\left(e^{j 2 \pi f}\right)}{S_{N}\left(e^{j 2 \pi f}\right)}\right) d f\right) \text {. }
$$

We make a few remarks and interpretations regarding the capacity-achieving predictive configuration, which further enhance its duality relationship with the predictive realization of the RDF.
Slicing and Coding We assumed that the decoder has access to past symbols. In the simplest realization, this is achieved by a decision element ("slicer") that works on a symbol-by-symbol basis. In practice however, to approach capacity, the slicer must be replaced by a "decoder". Here we must actually break with the assumption that $X_{n}$ is a Gaussian process. We implicitly assume that $X_{n}$ are symbols of a capacity- achieving AWGN code. The slicer should be viewed as a mnemonic aid where in practice an optimal decoder should be used.

However, we encounter two problems with this interpretation. First, the common view of a slicer is as a nearest neighbor quantizer. Thus in order to function correctly, the noise $E_{n}$ in (55) must be independent of the symbols $U_{n}$ and not of the estimator $V_{n}$ (i.e., the channel should be "forward" additive: $V_{n}=U_{n}+E_{n}$ ). This can be achieved by dithering the codebook via a modulo-shift as in [6]. This is reminiscent to the dithered quantization approach of Section VI Another difficulty is the conflict between the inherent decoding delay of a good code, and the sequential nature of the noiseprediction DFE configuration. Again (as with vector-DPCM in Section V, this may in principle be solved by incorporating an interleaver as suggested by Guess and Varanasi [11].

Capacity achieving shaping filter. For any spectral shaping filter $h_{1, n}$, the mutual information is given by (53). The shaping filter $h_{n}$ which maximizes the mutual information (and yields capacity) under the power constraint (51) is given by the parametric water-filling formula:

$$
\sigma_{U}^{2}\left|H_{1}\left(e^{j 2 \pi f}\right)\right|^{2}=\left[\theta-S_{N}\left(e^{j 2 \pi f}\right)\right]^{+},
$$

where the "water level" $\theta$ is chosen so that the power constraint is met with equality,

$$
\begin{aligned}
\sigma_{X}^{2} & =\int_{-1 / 2}^{1 / 2} \sigma_{U}^{2}\left|H\left(e^{j 2 \pi f}\right)\right|^{2} d f \\
& =\int_{-1 / 2}^{1 / 2}\left\lceil\theta-S_{N}\left(e^{j 2 \pi f}\right)\right\rceil^{+} d f=P .
\end{aligned}
$$

Using this choice, and arbitrarily setting

$$
\sigma_{U}^{2}=\theta
$$

it can be verified that the shaping filter $H_{1}\left(e^{j 2 \pi f}\right)$ and the estimation filter $H_{2}\left(e^{j 2 \pi f}\right)$ satisfy the same complex conjugate relation as the RDF-achieving pre- and post-filters (13) and (18)

$$
H_{2}\left(e^{j 2 \pi f}\right)=H_{1}^{*}\left(e^{j 2 \pi f}\right) \text {. }
$$


Under the same choice, we also have that:

$$
S_{D}\left(e^{j 2 \pi f}\right)=\min \left\{S_{N}\left(e^{j 2 \pi f}\right), \theta\right\} .
$$

Shaping, estimation and prediction at high SNR. At high signal-to-noise ratio (SNR), the shaping filter $H_{1}$ and the estimation filter $H_{2}$ become all-pass, and can be replaced by scalar multipliers. If we set the symbol variance as in 61, then we get at high SNR $\sigma_{U}^{2} \approx P$, so $X_{n} \approx U_{n}$ and $\hat{U}_{n} \approx Y_{n}$. It follows that the estimation error $D_{n} \approx N_{n}$, and therefore the slicer error $E_{n}$ becomes simply the prediction error (or the entropy power) of the channel noise $N_{n}$. This is the well known "zero-forcing DFE" solution for optimum detection at high SNR [1]. We shall next see that the same behavior of the slicer error holds even for non-asymptotic conditions.

The prediction process when the Shannon upper bound is tight. The Shannon upper bound (SUB) on capacity states that

$$
\begin{aligned}
C & \leq \frac{1}{2} \log \left(2 \pi e \sigma_{Y}^{2}\right)-\bar{h}(N) \\
& \leq \frac{1}{2} \log \left(\frac{P+\sigma_{N}^{2}}{P_{e}(N)}\right) \triangleq C_{\mathrm{SUB}}
\end{aligned}
$$

where

$$
\sigma_{N}^{2}=\int_{-1 / 2}^{1 / 2} S_{N}\left(e^{j 2 \pi f}\right) d f
$$

is the variance of the equivalent noise, and where equality holds if and only if the output $Y_{n}$ is white. This in turn is satisfied if and only if

$$
\theta \geq \max _{f} S_{N}\left(e^{j 2 \pi f}\right)
$$

in which case $\theta=P+\sigma_{N}^{2}$.

If we choose $\sigma_{U}^{2}$ according to (61), we have:

- The shaping and estimation filters satisfy

$$
\left|H_{1}\left(e^{j 2 \pi f}\right)\right|^{2}=\left|H_{2}\left(e^{j 2 \pi f}\right)\right|^{2}=1-\frac{S_{N}\left(e^{j 2 \pi f}\right)}{\theta} .
$$

- $U_{n}$ and $Y_{n}$ are white, with the same variance $\theta$.

- $X_{n}$ and $\hat{U}_{n}$ have the same power spectrum, $\theta-S_{N}\left(e^{j 2 \pi f}\right)$.

- The power spectrum of $D_{n}$ is equal to the power spectrum of the noise $N_{n}, S_{N}\left(e^{j 2 \pi f}\right)$. Consequently, the variance of $E_{n}$ which is equal to the entropy-power of $D_{n}$, is equal to $P_{e}(N)$.

- As a consequence we have

$$
\begin{aligned}
I\left(V_{n} ; V_{n}+E_{n}\right) & =h\left(U_{n}\right)-h\left(E_{n}\right) \\
& =h(\mathcal{N}(0, \theta))-h\left(\mathcal{N}\left(0, P_{e}(N)\right)\right) \\
& =\frac{1}{2} \log \left(\frac{P+\sigma_{N}^{2}}{P_{e}(N)}\right)
\end{aligned}
$$

which is indeed the SUB 63.

An alternative derivation of Theorem 3 in the spectral domain. Similarly to the alternative proof of Theorem 1 one can prove Theorem 3 using the spectra derived above.

\section{SUMMARY}

We demonstrated the dual role of prediction in ratedistortion theory of Gaussian sources and capacity of ISI channels. These observations shed light on the configurations of DPCM (for source compression) and FFE-DFE (for channel demodulation), and show that in principle they are "information lossless" for any distortion / SNR level. The theoretic bounds, RDF and capacity, can be approached in practice by appropriate use of feedback and linear estimation in the time domain combined with coding across the "spatial" domain.

A prediction-based system has in many cases a delay lower than that of a frequency domain approach, as is well known in practice. We slightly touched on this issue when discussing the 0.5 bit loss due to avoiding the ("non-causal") pre/post filters. But the full potential of this aspect requires further study.

It is tempting to ask whether the predictive form of the RDF can be extended to more general sources and distortion measures (and similarly for capacity of more general ISI channels). Yet, examination of the arguments in our derivation reveals that it is strongly tied to the quadratic-Gaussian case:

- The orthogonality principle, implied by the MMSE criterion, guarantees the whiteness of the noisy prediction error $Z q_{n}$ and its orthogonality with the past.

- Gaussianity implies that orthogonality is equivalent to statistical independence.

For other error criteria and/or non-Gaussian sources, prediction (either linear or non-linear) is in general unable to remove the dependence on the past. Hence the scalar mutual information over the prediction error channel would in general be greater than the mutual information rate of the source before prediction.

\section{ACKNOWLEDGEMENT}

We'd like to thank Robert M. Gray for pointing to us the origin of DPCM in a U.S. patent by C.C. Cutler in 1952.

\section{APPENDIX}

\section{A. Proof of Theorem 2}

It will be convenient to look at $K$-blocks, which we denote by bold letters as in Section VI Substituting the ECDQ rate definition (40) and the $K$-block directed information definition (45), the required result (46) becomes:

$$
H\left(\mathbf{Q}_{1}^{M} \mid \mathbf{D}_{1}^{M}\right)=\sum_{m=1}^{M} I\left(\mathbf{Z}^{m} ; \mathbf{Z} q_{m} \mid \mathbf{Z} q_{m}^{m-1}\right)
$$

Using the chain rule for entropies, it is enough to show that:

$$
H\left(\mathbf{Q}_{m} \mid \mathbf{Q}_{1}^{m-1}, \mathbf{D}_{1}^{M-1}\right)=I\left(\mathbf{Z} q_{m} ; \mathbf{Z}_{1}^{m} \mid \mathbf{Z} q_{1}^{m-1}\right)
$$


To that end, we have the following sequence of equalities:

$$
\begin{array}{ll} 
& H\left(\mathbf{Q}_{m} \mid \mathbf{Q}_{1}^{m-1}, \mathbf{D}_{1}^{M-1}\right) \\
\stackrel{(a)}{=} & H\left(\mathbf{Q}_{m} \mid \mathbf{Q}_{1}^{m-1}, \mathbf{D}_{1}^{m}\right) \\
\stackrel{(b)}{=} & H\left(\mathbf{Q}_{m} \mid \mathbf{Q}_{1}^{m-1}, \mathbf{D}_{1}^{m}\right)-H\left(\mathbf{Q}_{m} \mid \mathbf{Q}_{1}^{m-1}, \mathbf{Z}_{1}^{m}, \mathbf{D}_{1}^{m}\right) \\
= & I\left(\mathbf{Q}_{m} ; \mathbf{Z}_{1}^{m} \mid \mathbf{Q}_{1}^{m-1}, \mathbf{D}_{1}^{m}\right) \\
\stackrel{(\text { c })}{=} & I\left(\mathbf{Q}_{m}-\mathbf{D}_{m} ; \mathbf{Z}_{1}^{m} \mid \mathbf{Q}_{1}^{m-1}, \mathbf{D}_{1}^{m}\right) \\
= & I\left(\mathbf{Z}_{m} ; \mathbf{Z}_{1}^{m} \mid \mathbf{Q}_{1}^{m-1}, \mathbf{D}_{1}^{m}\right) \\
= & I\left(\mathbf{Z} q_{m} ; \mathbf{Z}_{1}^{m} \mid \mathbf{Q}_{1}^{m-1}-\mathbf{D}_{1}^{m-1}, \mathbf{D}_{1}^{m}\right) \\
= & I\left(\mathbf{Z} q_{m} ; \mathbf{Z}_{1}^{m} \mid \mathbf{Z} q_{1}^{m-1}, \mathbf{D}_{1}^{m}\right) \\
\stackrel{(d)}{=} & I\left(\mathbf{Z} q_{m} ; \mathbf{Z}_{1}^{m} \mid \mathbf{Z} q_{1}^{m-1}, \mathbf{D}_{m}\right) \\
= & I\left(\mathbf{Z} q_{m}, \mathbf{D}_{m} ; \mathbf{Z}_{1}^{m} \mid \mathbf{Z}_{1}^{m-1}\right)-I\left(\mathbf{D}_{m} ; \mathbf{Z}_{1}^{m} \mid \mathbf{Z} q_{1}^{m-1}\right) \\
\stackrel{(e)}{=} & I\left(\mathbf{Z} q_{m} ; \mathbf{Z}_{1}^{m} \mid \mathbf{Z} q_{1}^{m-1}\right)-I\left(\mathbf{D}_{m} ; \mathbf{Z}_{1}^{m} \mid \mathbf{Z} q_{1}^{m-1}\right) \\
\stackrel{(f)}{=} & I\left(\mathbf{Z} q_{m} ; \mathbf{Z}_{1}^{m} \mid \mathbf{Z} q_{1}^{m-1}\right) .
\end{array}
$$

In this sequence, equality (a) comes from the independent dither generation and causality of feedback. (b) is justified because $\mathbf{Q}_{m}$ is a deterministic function of the elements on which the subtracted entropy is conditioned, thus entropy is 0 . In (c) we subtract from the left hand side argument of the mutual information one of the variables upon which mutual information is conditioned. (d) and (e) hold since each dither vector $\mathbf{D}_{m}$ is a deterministic function of the corresponding quantizer output $\mathbf{Z} q_{m}$. Finally, (f) is true since $\mathbf{Z}_{1}^{m}$ is independent of $\mathbf{D}_{m}$ (both conditioned on past quantized values and unconditioned).

\section{REFERENCES}

[1] J. Barry, E. A. Lee and D. G. Messerschmitt. Digital Communication. Kluwer Academic Press, 2004 (third edition).

[2] T. Berger. Rate Distortion Theory: A Mathematical Basis for Data Compression. Prentice-Hall, Englewood Cliffs, NJ, 1971.

[3] J. Chen, C. Tian, T. Berger, and S. S. Hemami. Multiple Description Quantization via Gram-Schmidt Orthogonalization. IEEE Trans. Information Theory, IT-52:5197-5217, Dec. 2006.

[4] J.M. Cioffi, G.P. Dudevoir, M.V. Eyuboglu, and G.D. J. Forney. MMSE Decision-Feedback Equalizers and Coding - Part I: Equalization Results. IEEE Trans. Communications, COM-43:2582-2594, Oct. 1995.

[5] T. M. Cover and J. A. Thomas. Elements of Information Theory. Wiley, New York, 1991.

[6] U. Erez and R. Zamir. Achieving $\frac{1}{2} \log (1+S N R)$ on the AWGN channel with lattice encoding and decoding. IEEE Trans. Information Theory, IT-50:2293-2314, Oct. 2004.

[7] G. D. Forney, Jr. Shannon meets Wiener II: On MMSE estimation in successive decoding schemes. In 42st Annual Allerton Conference on Communication, Control, and Computing, Allerton House, Monticello, Illinois, Oct. 2004.

[8] R. G. Gallager. Information Theory and Reliable Communication. Wiley, New York, N.Y., 1968.

[9] A. Gersho and R. M. Gray. Vector Quantization and Signal Compression Kluwer Academic Pub., Boston, 1992.

[10] G.D.Gibson, T.Berger, T.Lookabaugh, D.Lindbergh, and R.L.Baker Digital Compression for Multimedia: Principles and Standards'. Morgan Kaufmann Pub., San Fansisco, 1998.

[11] T. Guess and M. K. Varanasi. An information-theoretic framework for deriving canonical decision-feedback receivers in gaussian channels. IEEE Trans. Information Theory, IT-51:173-187, Jan. 2005.

[12] N. S. Jayant and P. Noll. Digital Coding of Waveforms. Prentice-Hall, Englewood Cliffs, NJ, 1984.

[13] K. T. Kim and T. Berger. Sending a Lossy Version of the Innovations Process is Suboptimal in QG Rate-Distortion. In Proceedings of ISIT2005, Adelaide, Australia, pages 209-213, 2005.
[14] T. Linder and R. Zamir, Causal coding of stationary sources and individual sequences with high hesolution. IEEE Trans. Information Theory, IT-52:662-680, Feb. 2006.

[15] J. Massey. Causality, Feedback and Directed Information. In Proc. IEEE Int. Symp. on Information Theory, pages 303-305, 1990.

[16] R. Zamir and M. Feder. On universal quantization by randomized uniform / lattice quantizer. IEEE Trans. Information Theory, pages 428436, March 1992.

[17] R. Zamir and M. Feder. On lattice quantization noise. IEEE Trans. Information Theory, pages 1152-1159, July 1996.

[18] R. Zamir and M. Feder. Information rates of pre/post filtered dithered quantizers. IEEE Trans. Information Theory, pages 1340-1353, September 1996. 